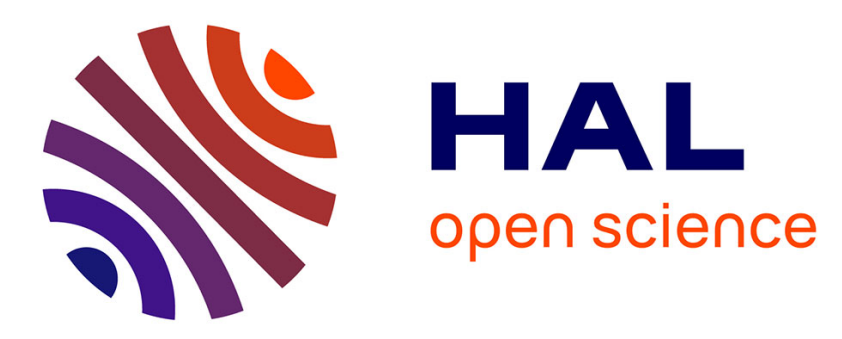

\title{
Acceleration of the imaginary time method for spectrally computing the stationary states of Gross-Pitaevskii equations
}

Xavier Antoine, Christophe Besse, Romain Duboscq, Vittorio Rispoli

\section{To cite this version:}

Xavier Antoine, Christophe Besse, Romain Duboscq, Vittorio Rispoli. Acceleration of the imaginary time method for spectrally computing the stationary states of Gross-Pitaevskii equations. Computer Physics Communications, 2017, 219, pp.70-78. 10.1016/j.cpc.2017.05.008 . hal-01356227

\section{HAL Id: hal-01356227 \\ https://hal.science/hal-01356227}

Submitted on 25 Aug 2016

HAL is a multi-disciplinary open access archive for the deposit and dissemination of scientific research documents, whether they are published or not. The documents may come from teaching and research institutions in France or abroad, or from public or private research centers.
L'archive ouverte pluridisciplinaire HAL, est destinée au dépôt et à la diffusion de documents scientifiques de niveau recherche, publiés ou non, émanant des établissements d'enseignement et de recherche français ou étrangers, des laboratoires publics ou privés. 


\title{
Acceleration of the imaginary time method for spectrally computing the stationary states of Gross-Pitaevskii equations
}

\author{
Xavier ANTOINE*,a, Christophe BESSE ${ }^{\mathrm{b}}$, Romain DUBOSCQ ${ }^{\mathrm{b}}$, Vittorio RISPOLI ${ }^{\mathrm{b}}$ \\ ${ }^{a}$ Institut Elie Cartan de Lorraine, Université de Lorraine, Inria Nancy-Grand Est, SPHINX team, F-54506 \\ Vandoeuvre-lès-Nancy Cedex, France. \\ ${ }^{b}$ Université Paul Sabatier, Institut des Mathématiques de Toulouse, France.
}

\begin{abstract}
The aim of this paper is to propose a simple accelerated spectral gradient flow formulation for solving the Gross-Pitaevskii Equation (GPE) when computing the stationary states of Bose-Einstein Condensates. The new algorithm, based on the recent iPiano minimization algorithm [35], converges three to four times faster than the standard implicit gradient scheme. To support the method, we provide a complete numerical study for 1d-2d-3d GPEs, including rotation and dipolar terms.
\end{abstract}

Keywords: Bose-Einstein condensation; rotating Gross-Pitaevskii equation; stationary states; imaginary time formulation; accelerated gradient; pseudospectral approximation.

\section{Introduction}

At temperatures $T$ which are smaller than the critical temperature $T_{\mathrm{c}}$, the Gross-Pitaevskii equation (GPE) can be used to model the behavior of Bose-Einstein Condensates (BECs). The first experimental realization of BECs was in 1995 [4, 8, 16, 23, 26] while they were theoretically predicted seventy years before by S.N. Bose and A. Einstein. This state of matter leads to the possibility of studying quantum physics at the macroscopic scale. Later, the nucleation of quantum vortices was observed [1, 17, 30, 31, 32, 36, 40 leading to the understanding of such BECs, modeled by the rotating GPE. More precisely, for a given initial data $\psi(t=0, \mathbf{x})=\psi_{0}(\mathbf{x})$, a rotating BEC is represented by a wave function $\psi(t, \mathbf{x})$ solution to the dimensionless time-dependent GPE [8, 11]

$$
i \partial_{t} \psi(t, \mathbf{x})=-\frac{1}{2} \Delta \psi(t, \mathbf{x})+V(\mathbf{x}) \psi(t, \mathbf{x})+\beta|\psi(t, \mathbf{x})|^{2} \psi(t, \mathbf{x})-\boldsymbol{\Omega} \cdot \mathbf{L} \psi(t, \mathbf{x})
$$

for $\mathbf{x} \in \mathbb{R}^{d}, d=1,2,3, t>0$ and $\Delta:=\sum_{j=1}^{d} \partial_{x_{j}}^{2}$. The function $V$ is the confining potential. The parameter $\beta$ is the nonlinearity strength that describes the (attractive or repulsive) interactions between atoms within the condensate. Essentially, we consider a cubic nonlinearity but the case of dipolar gases [8, 11] is also analyzed at the end of the paper to show that our contribution is general. In order to obtain the nucleation of vortices [1, 17, 30, 31, 32, 36, 40, the following rotating term is added

$$
\boldsymbol{\Omega} \cdot \mathbf{L}=\Omega L_{z}=-i \Omega\left(x \partial_{y}-y \partial_{x}\right),
$$

assuming $\boldsymbol{\Omega}=(0,0, \Omega)^{t}, \Omega$ being called the rotational velocity.

In this paper, we wish to compute the stationary states of the GPE, i.e. a solution of the form

$$
\psi(t, \mathbf{x})=e^{-i \mu t} \phi(\mathbf{x})
$$

where $\mu$ is the chemical potential and $\phi$ is a function that only depends on $\mathbf{x}$. By using equation (1), we deduce a nonlinear elliptic equation which reads as

$$
\mu \phi(\mathbf{x})=-\frac{1}{2} \Delta \phi(\mathbf{x})+V(\mathbf{x}) \phi(\mathbf{x})+\beta|\phi(\mathbf{x})|^{2} \phi(\mathbf{x})-\Omega L_{z} \phi(\mathbf{x}),
$$

\footnotetext{
${ }^{*}$ Corresponding author
} 
under the normalization constraint

$$
\|\phi\|_{0}^{2}=\int_{\mathbb{R}^{d}}|\phi(\mathbf{x})|^{2} d \mathbf{x}=1,
$$

where $\|\cdot\|_{0}$ is the $L^{2}\left(\mathbb{R}^{d}\right)$-norm. If $\phi$ is given, we can compute directly $\mu$ through

$$
\mu_{\beta, \Omega}(\phi)=\mathscr{E}_{\beta, \Omega}(\phi)+\frac{\beta}{2} \int_{\mathbb{R}^{d}}|\phi(\mathbf{x})|^{4} d \mathbf{x},
$$

by defining the energy $\mathscr{E}_{\beta, \Omega}$ as

$$
\mathscr{E}_{\beta, \Omega}(\phi)=\int_{\mathbb{R}^{d}} \frac{1}{2}|\nabla \phi|^{2}+V|\phi|^{2}+\frac{\beta}{2}|\phi|^{4}-\Omega \Re\left(\phi^{*} L_{z} \phi\right) d \mathbf{x},
$$

where $\phi^{*}$ is the complex conjugate function of $\phi$. Another interpretation is to say that eigenfunctions are the critical points of the energy functional $\mathscr{E}_{\beta, \Omega}$ over the unit sphere: $\mathbb{S}:=\left\{\|\phi\|_{0}=1\right\}$. Then, (4) can be seen as the Euler-Lagrange equation related to the minimization problem of the energy functional under constraint [13].

Various numerical methods [8, 11] have been designed to compute the stationary states of GPEs, most particularly to get the ground state and the excited state solutions. It is admitted that deriving robust and efficient numerical approaches for the stationary state computation is difficult, most particularly when the nonlinearity is large and the rotation velocity is high. More generally, methods are based either on solving the nonlinear eigenvalue problem [27, 37, 38] or deriving nonlinear optimization techniques under constraints [14, 18, 19, 24, 25. A standard alternative approach is the Imaginary Time Method (ITM) also called Continuous Normalized Gradient Flow (CNGF) [2, 6, 8, 11, 13, 15, 21, 22, 41. In this paper we propose a modified version of the so-called BESP (Backward Euler pseudoSPectral) scheme for the CNGF that is known to be well-adapted to the computation of steady state solutions. Starting from the standard imaginary time method, we speed up its convergence using accelerated gradient techniques 20, 33, 34. We exploit the recent iPiano method [35], proven to be effective in other contexts like image processing. Starting from its definition, we develop a new implicit version which is more adapted to our purposes and only requires almost no modification of BESP. We thus put together the benefits of both the common BESP scheme and acceleration techniques. The resulting scheme is much more efficient than the initial BESP scheme. It is also more robust and has better stability properties than directly appliying the iPiano algorithm. Our numerical study in $1 \mathrm{~d}-2 \mathrm{~d}-3 \mathrm{~d}$ for nontrivial situations shows that the gain in efficiency is really important for a simple modification of BESP. Let us remark that this general idea may be applicable to other recent techniques for computing the stationary states, like for example for the regularized Newton method proposed in 39.

The plan of the paper is the following. In Section 2, we first present the imaginary time method and its CNGF formulation. This allows us to next introduce a well-adapted accelerated formulation of the CNGF formulation by adapting the Backward Euler scheme and the iPiano method. The pseudospectral discretization in space is then given. In Section 3, we present a thorough numerical study for $1 \mathrm{~d}, 2 \mathrm{~d}$ and $3 \mathrm{~d}$ problems, with rotational term and for dipolar gases. This study shows that the new accelerated method provides an important gain in terms of computational efficiency, for a slight modification of the BESP scheme. Finally, we conclude in Section 4

\section{Accelerating the imaginary time method}

\subsection{Imaginary time method: standard gradient-type schemes}

The Imaginary Time Method (ITM) is a commonly used method in the physics literature to compute the stationary state solutions to the GPE [2, 3, 15, 13, 21, 22, 28, 29. The approach consists in obtaining a reformulation of the Equation (4) as a complex time-dependent GPE. This also corresponds to the evolution of the gradient flow with discrete normalization (GFDN). Fixing uniformly sampled discrete times $0=t^{0}<t^{1}<\ldots<t^{n}<\ldots$ (with time step $\delta t>0$ ), the ITM leads to solving, in each time interval $\left[t^{n}, t^{n+1}\right]$, the gradient flow and then projecting the solution (i.e. $L^{2}$-normalization). The resulting algorithm writes

$$
\left\{\begin{aligned}
\partial_{t} \phi(t, x) & \left.=-\nabla_{\phi^{*}} \mathscr{E}_{\beta, \Omega}(\phi)=\frac{1}{2} \Delta \phi-V \phi-\beta|\phi|^{2} \phi+\Omega L_{z} \phi, \quad t \in\right] t^{n}, t^{n+1}[ \\
\phi\left(t^{n+1}, \mathbf{x}\right) & =\lim _{t \rightarrow t^{n+1}} \frac{\phi(t, \mathbf{x})}{\|\phi(t, \mathbf{x})\|_{0}}
\end{aligned}\right.
$$


From the mathematical point of view, the ITM has been studied for the non rotating case in [13] by being written as a Conjugate Normalized Gradient Flow (CNGF) formulation. In particular, the authors show that the time discretization of the CNGF must be carefully considered. They prove that the semi-implicit Backward Euler scheme is particularly well-adapted since it indeed leads to an energy diminishing formulation without any constraint on the time step $\delta t$ for the linear operator. Concretely, being given an initial state $\phi^{0}$, the semi-implicit Backward Euler (BE) time discretization of the ITM leads to: $\forall n \in \mathbb{N}$, compute $\phi^{n+1}$ such that

$$
\left\{\begin{array}{l}
\phi_{0}=\phi^{n}-\delta t \nabla_{\phi^{*}} \tilde{\mathscr{E}}_{\beta, \Omega}\left(\phi^{n}, \phi_{0}\right), \\
\phi^{n+1}=\frac{\phi_{0}}{\left\|\phi_{0}\right\|_{0}}
\end{array}\right.
$$

where

$$
\nabla_{\phi^{*}} \tilde{\mathscr{E}}_{\beta, \Omega}(u, w)=\frac{1}{2} \Delta w-V w-\beta|u|^{2} w+\Omega L_{z} w
$$

The semi-implicit feature of the scheme appears in the nonlinear term.

If a Forward Euler (FE) scheme is used, then a CFL-like stability constraint appears on $\delta t[13$ ] and involves the extrema of the potential, affecting hence the robustness of the scheme (as for the CrankNicolson scheme for the ITM). The resulting linear scheme then writes: $\forall n \in \mathbb{N}$, compute $\phi^{n+1}$ such that

$$
\left\{\begin{array}{l}
\phi_{0}=\phi^{n}-\delta t \nabla_{\phi^{*}} \mathscr{E}_{\beta, \Omega}\left(\phi^{n}\right) \\
\phi^{n+1}=\frac{\phi_{0}}{\left\|\phi_{0}\right\|_{0}}
\end{array}\right.
$$

which is nothing else than a standard (explicit) projected gradient in terms of optimization method. This is a difference with the BE scheme (9) which rather consists in an implicit version of the projected gradient method with a linearization step. The time step $\delta t$ is then the step of the gradient method.

\subsection{Imaginary time method: accelerated gradient-type schemes}

Accelerated gradients schemes were first introduced by Nesterov in the eighties 33, 34. The main idea behind the acceleration of the gradient is to add a momentum term in the gradient flow. For our problem, since we wish to compute a stationary state of the GPE with a mass constraint, we need to include an extra $L^{2}$-normalization step into the algorithm. An important feature of the accelerated gradient-type methods is that their efficiency and stability strongly depends on some tuning parameters. Improvements and extensions of the accelerated gradient schemes can be found e.g. in [20. We extensively tested such approaches in our context with both the explicit/semi-implicit implementation of the gradient terms. Despite the apparent improvement in terms of efficiency of these methods to reach a given state, it turns out that they are usually unstable in the context of the GPE. Indeed, there is no clear rule to fix the tuning parameters. Therefore, the efficiency/robustness of the corresponding accelerated scheme can be easily lost.

Nevertheless, among all these techniques, a class of accelerated gradient-type methods appears to be stable for our problem, i.e. the resulting efficiency is stable for a well-chosen tuning parameter that weakly depends on the physical/discretization problem parameters. The very recent method, called iPiano, was presented in 35] for computer vision problems (image denoising). The aim of the present paper is 1) to propose a stable adaptation of the iPiano method to the computation of the stationary states of the GPE and 2) to confirm its efficiency for the 1d-2d-3d rotating GPE when a spectral spatial discretization is used.

The iPiano method is a minimization technique which is convergent when the objective function is the sum of a non-convex smooth function and a convex non-smooth function. More explicitly, the class of non-convex problems that can be solved writes down as

$$
\min _{\mathbf{x} \in \mathbb{R}^{p}} f(\mathbf{x})+g(\mathbf{x})
$$

where $\mathrm{g}$ is a convex (possibly non-smooth) function and $f$ is a smooth (possibly non-convex) function, from $\mathbb{R}^{p}$ onto $\mathbb{R}$. The scheme then produces a sequence $\left(\mathbf{x}_{n}\right)_{n \in \mathbb{N}}$ which converges to the solution of the minimization problem and given by the following iterative procedure

$$
\mathbf{x}^{n+1}=(I-\alpha \partial g)^{-1}\left(\mathbf{x}^{n}-\alpha \nabla f\left(\mathbf{x}^{n}\right)+\beta_{\text {iPiano }}\left(\mathbf{x}^{n}-\mathbf{x}^{n-1}\right)\right)
$$


where $\alpha>0$ and $0 \leq \beta_{\text {iPiano }}<1$ are respectively the step size and the inertial parameter. The inertial force term $\beta_{\text {iPiano }}\left(\mathbf{x}^{n}-\mathbf{x}^{n-1}\right)$ ensures the acceleration of the scheme. In Eq. [12), we directly remark that the method is explicit for the non-convex part of the objective function but implicit for the non-smooth part via a proximal map. For the computation of the stationary states of the GPE, the energy functional is smooth. Therefore, we consider the following functions $f$ and $g$ in our situation

$$
f(\phi)=\mathscr{E}_{\beta, \Omega}(\phi) \quad \text { and } \quad g(\phi)=0,
$$

leading to the scheme

$$
\left\{\begin{array}{l}
\phi_{0}=\phi^{n}-\delta t \nabla_{\phi^{*}} \mathscr{E}_{\beta, \Omega}\left(\phi^{n}\right)+\beta_{\mathrm{iPiano}}\left(\phi^{n}-\phi^{n-1}\right) \\
\phi^{n+1}=\frac{\phi_{0}}{\left\|\phi_{0}\right\|_{0}}
\end{array}\right.
$$

where $\alpha=\delta t$. With $g=0$, the iPiano scheme is usually called the Heavy-ball method. This indeed corresponds to an explicit finite difference scheme of the well-known Heavy-ball friction dynamical system

$$
\ddot{\phi}(t)+\gamma \dot{\phi}(t)+\nabla f(\phi(t))=0
$$

which should be compared to the usual gradient flow approach

$$
\dot{\phi}(t)+\nabla f(\phi(t))=0 .
$$

Hence, applying the iPiano technique can be interpreted as an accelerated explicit gradient method for (11) that reduces to 11 for $\beta_{\text {iPiano }}=0$. Based on this remark, it is clear that the explicit scheme (13) inherits the stability problems from the explicitation of the gradient. This is even true when a costly optimal time step procedure is used to adapt $\alpha(=\delta t)$. To overcome this problem, we propose to use a semi-implicit version of iPiano in the spirit of the BE scheme (9). Therefore, the resulting scheme writes: $\forall n \in \mathbb{N}$, compute $\phi^{n+1}$ such that

$$
\left\{\begin{array}{l}
\phi_{0}=\phi^{n}-\delta t \nabla_{\phi^{*}} \tilde{\mathscr{E}}_{\beta, \Omega}\left(\phi^{n}, \phi_{0}\right)+\beta_{\mathrm{iPiano}}\left(\phi^{n}-\phi^{n-1}\right), \\
\phi^{n+1}=\frac{\phi_{0}}{\left\|\phi_{0}\right\|_{0}},
\end{array}\right.
$$

where $\phi^{0}$ is a given initial data, $\delta t$ is the time step, $\beta_{\text {iPiano }} \in[0,1)$ is the inertial parameter and $\phi^{-1}=\phi^{0}$. In practice we separate the first line of the algorithm 14 into the two following steps: $\forall n \in \mathbb{N}$, compute $\phi^{n+1}$ such that

$$
\left\{\begin{array}{l}
\phi_{0}=\phi^{n}+\beta_{\mathrm{iPiano}}\left(\phi^{n}-\phi^{n-1}\right) \\
\phi_{1}=\phi_{0}-\delta t \nabla_{\phi^{*}} \tilde{E}_{\beta, \Omega}\left(\phi_{0}, \phi_{1}\right) \\
\phi^{n+1}=\frac{\phi_{1}}{\left\|\phi_{1}\right\|_{0}}
\end{array}\right.
$$

Trivially, $\beta_{\text {iPiano }}=0$ corresponds to the BE scheme $(9)$ which lets to think that the resulting scheme is much more stable than (13). In terms of implementation and computational cost, the scheme (15) requires almost no additional effort compared with $\mathrm{BE}$. Concerning the parameter $\beta_{\text {iPiano }}$, there is no explicit formula to determine its optimal value which is supposed to be problem dependent. Nevertheless, the extensive numerical study in Section 3 shows that an almost optimal value corresponds to $\beta_{\text {iPiano }} \simeq$ $0.75-0.8$ and is weakly sensitive to the physical and discretization parameters. This value which is obtained for our specific problem seems to be more general for the application range since for instance in [35] a nearly optimal value seems to be $\beta_{\text {iPiano }} \simeq 0.8$ for problems in image processing.

\subsection{Pseudo-spectral discretization in space}

To correctly capture the nucleation of vortices, high-order discretizations in space are needed. Highorder finite difference or finite element schemes could be used with e.g. adaptive mesh techniques. However, in some situations like for dipolar interactions [11, 12, or multi-components BECs [9, 10, 11, these approaches may be complicate to implement and lead to huge computational costs. The direction that we follow here uses pseudo-spectral (SP) discretization techniques based on the Fast 
Fourier Transform (FFT) [5, 7, 11. We present now this approach in the two-dimensional case, the extension to the $1 \mathrm{~d}$ and $3 \mathrm{~d}$ cases being direct.

We introduce the spatial bounded computational domain $\mathcal{D}=]-a_{x} ; a_{x}[\times]-a_{y} ; a_{y}[$. The physics of BECs modeled by the GPE evolves inside the finite box $\mathcal{D}$. This naturally lets us the possibility to enforce periodic boundary conditions on $\phi$ at the boundary $\partial \mathcal{D}$ (even if homogeneous Dirichlet or Neumann boundary conditions could also be imposed). We introduce now the discrete grid with uniformly sampled points: $\mathcal{D}_{N_{x} N_{y}}:=\left\{\mathbf{x}_{k_{1}, k_{2}}=\left(x_{k_{1}}, y_{k_{2}}\right)\right\}_{\left(k_{1}, k_{2}\right) \in \mathcal{O}_{N_{x} N_{y}}}$, where

$$
\mathcal{O}_{N_{x} N_{y}}:=\left\{\left(k_{1}, k_{2}\right) \in \mathbb{N}^{2} \text { such that } k_{1}=0, \ldots, N_{x}-1, k_{2}=0, \ldots, N_{y}-1\right\}
$$

for $N_{x}$ and $N_{y}$ two even positive integers. We define

$$
x_{k_{1}+1}-x_{k_{1}}=h_{x}=2 a_{x} / N_{x}, \quad y_{k_{2}+1}-y_{k_{2}}=h_{y}=2 a_{y} / N_{y} .
$$

Let $\boldsymbol{\zeta}_{p q}=\left(\xi_{p}, \eta_{q}\right)$, with $\xi_{p}=p \pi / a_{x},-N_{x} / 2 \leq p \leq N_{x} / 2-1$, and $\eta_{q}=q \pi / a_{y},-N_{y} / 2 \leq q \leq N_{y} / 2-1$, be the associated discrete Fourier frequencies. The partial Fourier pseudo-spectral approximations $\tilde{\phi}$ of the function $\phi$ in the $x$ - and $y$-directions are then

$$
\widetilde{\phi}(t, x, y)=\frac{1}{N_{x}} \sum_{p=-N_{x} / 2}^{N_{x} / 2-1} \widehat{\widetilde{\phi}}_{p}(t, y) e^{i \xi_{p}\left(x+a_{x}\right)}, \quad \widetilde{\phi}(t, x, y)=\frac{1}{N_{y}} \sum_{q=-N_{y} / 2}^{N_{y} / 2-1} \widehat{\widetilde{\phi}}_{q}(t, x) e^{i \eta_{q}\left(y+a_{y}\right)},
$$

where $\widehat{\widetilde{\phi}}_{p}$ and $\widehat{\widetilde{\phi}}_{q}$ respectively designate the Fourier coefficients in the $x$ - and $y$-directions

$$
\widehat{\widetilde{\phi}_{p}}(t, y)=\sum_{k_{1}=0}^{N_{x}-1} \widetilde{\phi}_{k_{1}}(t, y) e^{-i \xi_{p}\left(x_{k_{1}}+a_{x}\right)}, \quad \widehat{\widetilde{\phi}}_{q}(t, x)=\sum_{k_{2}=0}^{N_{y}-1} \widetilde{\phi}_{k_{2}}(t, x) e^{-i \eta_{q}\left(y_{k_{2}}+a_{y}\right)} .
$$

The following notations are used: $\widetilde{\phi}_{k_{1}}(t, y)=\widetilde{\phi}\left(t, x_{k_{1}}, y\right)$ and $\widetilde{\phi}_{k_{2}}(t, x)=\widetilde{\phi}\left(t, x, y_{k_{2}}\right)$. To evaluate the operators, we define the matrices $\mathbb{I}, \mathbb{V}$ and $[[f(\phi)]]$ that apply pointwise in the physical space

$$
\mathbb{I}_{k_{1}, k_{2}}:=\delta_{k_{1}, k_{2}}, \quad \quad \mathbb{V}_{k_{1}, k_{2}}:=V\left(\mathbf{x}_{k_{1}, k_{2}}\right), \quad[[f(\phi)]]_{k_{1}, k_{2}}=f\left(\phi_{k_{1}, k_{2}}\right),
$$

for $\left(k_{1}, k_{2}\right) \in \mathcal{O}_{N_{x} N_{y}}$. The symbol $\delta_{k_{1}, k_{2}}$ is the Dirac delta symbol which is equal to 1 if and only if $k_{1}=k_{2}$ and 0 otherwise. We also define the four operators $\left[\left[\partial_{x}^{2}\right]\right]$, $\left[\left[\partial_{y}^{2}\right]\right], y\left[\left[\partial_{x}\right]\right]$ and $x\left[\left[\partial_{y}\right]\right]$ which are applied to $\widetilde{\phi}$ that approximates the continuous function $\phi$ for $\left(k_{1}, k_{2}\right) \in \mathcal{O}_{N_{x} N_{y}}$

$$
\begin{aligned}
& \partial_{x}^{2} \phi\left(\mathbf{x}_{k_{1}, k_{2}}\right) \approx\left(\left[\left[\partial_{x}^{2}\right]\right] \widetilde{\phi}\right)_{k_{1}, k_{2}}:=-\frac{1}{N_{x}} \sum_{p=-N_{x} / 2}^{N_{x} / 2-1} \xi_{p}^{2} \widehat{\left(\widetilde{\phi}_{k_{2}}\right)_{p}} e^{i \xi_{p}\left(x_{k_{1}}+a_{x}\right)}, \\
& \partial_{y}^{2} \phi\left(\mathbf{x}_{k_{1}, k_{2}}\right) \approx\left(\left[\left[\partial_{y}^{2}\right]\right] \widetilde{\phi}\right)_{k_{1}, k_{2}}:=-\frac{1}{N_{y}} \sum_{q=-N_{y} / 2}^{N_{y} / 2-1} \eta_{q}^{2} \widehat{\left(\widetilde{\phi_{k_{1}}}\right)_{q}} e^{i \eta_{q}\left(y_{k_{2}}+a_{y}\right)}, \\
& \left(x \partial_{y} \phi\right)\left(\mathbf{x}_{k_{1}, k_{2}}\right) \approx\left(x\left[\left[\partial_{y}\right]\right] \widetilde{\phi}\right)_{k_{1}, k_{2}}:=\frac{1}{N_{y}} \sum_{q=-N_{y} / 2}^{N_{y} / 2-1} i x_{k_{1}} \eta_{q} \widehat{\left(\widetilde{\phi}_{k_{1}}\right)_{q}} e^{i \eta_{q}\left(y_{k_{2}}+a_{y}\right)}, \\
& \left(y \partial_{x} \phi\right)\left(\mathbf{x}_{k_{1}, k_{2}}\right) \approx\left(y\left[\left[\partial_{x}\right]\right] \widetilde{\phi}\right)_{k_{1}, k_{2}}:=\frac{1}{N_{x}} \sum_{p=-N_{x} / 2}^{N_{x} / 2-1} i y_{k_{2}} \xi_{p} \widehat{\left(\widetilde{\phi}_{k_{2}}\right)_{p}} e^{i \xi_{p}\left(x_{k_{1}}+a_{x}\right)} .
\end{aligned}
$$

We introduce the discrete operators acting from $\mathbb{C}^{M}$ to $\mathbb{C}$, with $M=N_{x} N_{y}$ (in $2 \mathrm{~d}$ ), by

$$
[[\Delta]]:=\left[\left[\partial_{x}^{2}\right]\right]+\left[\left[\partial_{y}^{2}\right]\right], \quad\left[\left[\mathbb{L}_{z}\right]\right]:=-i\left(x\left[\left[\partial_{y}\right]\right]-y\left[\left[\partial_{x}\right]\right]\right) .
$$

The matrix $[[\Delta]]$ is diagonal in the Fourier space but not $\mathbb{L}_{z}$. We set

$$
\phi:=\left(\tilde{\phi}\left(\mathbf{x}_{k_{1}, k_{2}}\right)\right)_{\left(k_{1}, k_{2}\right) \in \mathcal{O}_{N_{x} N_{y}}}
$$

as the discrete unknown vector in $\mathbb{C}^{M}$. For the sake of conciseness, we do not make any distinction between an array $\phi$ in $\mathcal{M}_{N_{x} \times N_{y}}(\mathbb{C})$ (storage according to the $2 \mathrm{~d}$ grid) and the corresponding reshaped column vector in $\mathbb{C}^{M}$. We denote by $\mathcal{M}_{N_{x} \times N_{y}}(\mathbb{C})$ the vector space of $2 \mathrm{~d}$ complex-valued arrays. 
Let us introduce the complex-valued matrix

$$
\mathbb{A}_{\beta, \Omega}^{n}=\frac{1}{2}[[\Delta]]-[[V]]-\beta\left[\left[\left|\phi^{n}\right|^{2}\right]\right]+\Omega\left[\left[L_{z}\right]\right] .
$$

Applying the pseudo-spectral approximation scheme, system 15 writes down

$$
\left\{\begin{array}{l}
\phi_{0}=\phi^{n}+\beta_{\text {iPiano }}\left(\phi^{n}-\phi^{n-1}\right), \\
\left(\mathbb{I}-\delta t \mathbb{A}_{\beta, \Omega}^{n}\right) \phi_{1}=\phi_{0} \\
\phi^{n+1}=\frac{\phi_{1}}{\left\|\phi_{1}\right\|_{0}}
\end{array}\right.
$$

where $\|\phi\|_{0}$ is the discrete $L^{2}$-norm of a complex-valued vector field $\phi$ and the initial data $\phi^{-1,0}$ is fixed by evaluating $\phi^{-1,0}$ on the spatial grid $\mathcal{D}_{N_{x} N_{y}}$. The resulting scheme given in (18) is called $\operatorname{BESP}\left(\beta_{\text {iPiano }}\right)$. The stopping criterion for the convergence of the minimization procedure is based on the residual computed for the discrete $L^{\infty}$-norm between two successive iterates, i.e.

$$
\left\|\phi^{n}-\phi^{n+1}\right\|_{\infty}<\tau \delta t
$$

where $\|\phi\|_{\infty}:=\sup _{\left(k_{1}, k_{2}\right) \in \mathcal{O}_{N_{x} N_{y}}}\left|\phi\left(\mathbf{x}_{k_{1}, k_{2}}\right)\right|$ and the small value of $\tau$ is a priori fixed by the user. Solving (18) requires to iterate on $n$ sufficiently so that 19 is fulfilled. However, each iteration needs the solution to a complex-valued linear system with matrix $\left(\mathbb{I}-\delta t \mathbb{A}_{\beta, \Omega}^{n}\right)$. To obtain the solution to the linear system in (18) and since we do not have access explicitly to the matrix $\left[\left[A_{\beta, \Omega}^{n}\right]\right]$, we use the BiCGStab iterative solver which only requires some matrix-vector products evaluations that are the main computational contributions for one iteration of BiCGStab (with a computational cost related to the 1d FFT evaluations). In addition, the acceleration of the BiCGStab is realized through the (Fourier) diagonal Laplace preconditioner $(\mathbb{I}-\delta t[[\Delta]] / 2)^{-1}$ in the spirit of $[6]$. All along the paper, the convergence criterion of the preconditioned BiCGStab is fixed to $10^{-14}$ since we are using a spectral approximation. Let us call $N^{\text {out }}$ the number of outer iterations of the $\operatorname{BESP}\left(\beta_{\text {iPiano }}\right)$ algorithm 18 ) to converge according to $(19)$, with given $\beta, \Omega, \beta_{\text {iPiano }}$ and $\tau$. Each outer iteration requires $N_{n}^{\text {in }}$ inner iterations of the preconditioned BiCGStab to converge (with a residual equal to $10^{-14}$ ). As a consequence, a suitable way to understand the improved efficiency of $\operatorname{BESP}\left(\beta_{\text {iPiano }}\right)$ is to represent the total number

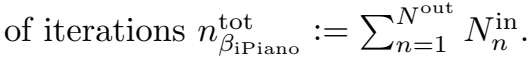

\section{Numerical results}

\subsection{The one-dimensional case}

We first analyze $\operatorname{BESP}\left(\beta_{\text {iPiano }}\right)$ for the $1 d$ case, i.e. without rotational term. The GPE involves a cubic nonlinearity and 1) a quadratic potential or 2) a quadratic-plus-quartic potential. The reference solution $\phi^{\text {ref }}$ is based on $\operatorname{BESP}(0)$ with $N_{x}=2^{12}$ and $\delta t=10^{-4}$. This allows us to study the accuracy of the method and most particularly the fact that the method converges towards the correct stationary state $\phi^{\text {computed }}$ through the error between the reference energy and the computed energy for $\operatorname{BESP}\left(\beta_{\mathrm{iPiano}}\right)$ given by

$$
\delta \mathscr{E}_{\beta, \Omega}:=\left|\mathscr{E}_{\beta, \Omega}\left(\phi^{\text {ref }}\right)-\mathscr{E}_{\beta, \Omega}\left(\phi^{\text {computed }}\right)\right| .
$$

To measure the gain of efficiency, we represent the ratio $r_{\beta_{\text {iPiano }}}:=n_{\beta_{\text {iPiano }}}^{\text {tot }} / n_{0}^{\text {tot }}(\%)$.

Let us consider the harmonic potential $V(x)=x^{2} / 2$. The numerical parameters are the following: $a_{x}=30, N_{x}=2^{10}, \delta t=10^{-2}, \tau=10^{-10}$ and $\beta \in[0,3000]$. We report on Figure 1 (left) the error $\delta \mathscr{E}_{\beta, 0}$ between the energy of the reference and computed solutions. Since the difference on the energy is uniformly small, we deduce that the $\operatorname{BESP}\left(\beta_{\mathrm{iPiano}}\right)$ scheme leads to the correct solution as long as $\beta_{\text {iPiano }}$ is not too close from 1 , which can also be checked on the stationary states computation. In Figure 1 (right), we see that $\operatorname{BESP}\left(\beta_{\text {iPiano }}\right)$ leads to an improved convergence with a nearly optimal value of

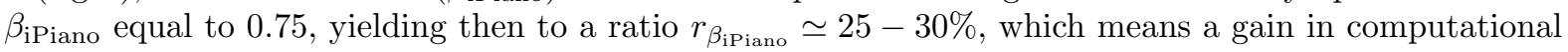
cost of about $70-75 \%$. This value is independent of the nonlinearity strength $\beta$.

Let us now analyse the sensitivity of $\operatorname{BESP}\left(\beta_{\text {iPiano }}\right)$ regarding the discretization parameters in time $\delta t$ and space $N_{x}$. Numerical simulations show that there is an optimal set of parameters $\left(\beta_{\mathrm{iPiano}}^{*}, \delta t^{*}\right)$ (see Figure 2, left) which reduces drastically the total number $n_{\beta_{\text {iPiano }}}^{\text {tot }}$ of BiCGStab iterations. We can infer $\left(\beta_{\mathrm{iPiano}}^{*}, \delta t^{*}\right) \in\left(10^{-3}, 10^{-2}\right) \times(0.7,0.9)$. Indeed, if the time step $\delta t$ is not small enough, then the 

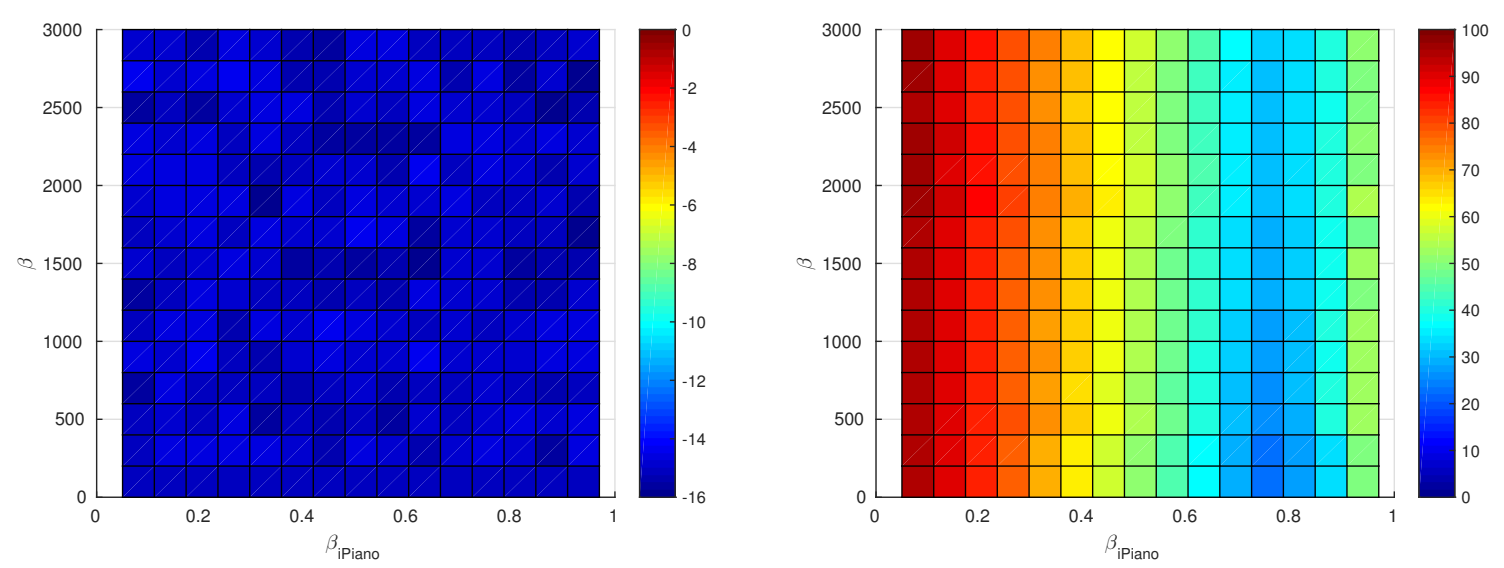

Figure 1: $\operatorname{BESP}\left(\beta_{\mathrm{iPiano}}\right)$ for the $1 \mathrm{~d}$ harmonic potential: error $\delta_{\mathscr{E}_{\beta, 0}}$ (left figure, $\log _{10}$ scale) and gain $r_{\beta_{\mathrm{iPiano}}}$ (right figure, $\%)$ vs. the inertial parameter $\beta_{\mathrm{iPiano}}$ and nonlinearity strength $\beta$.
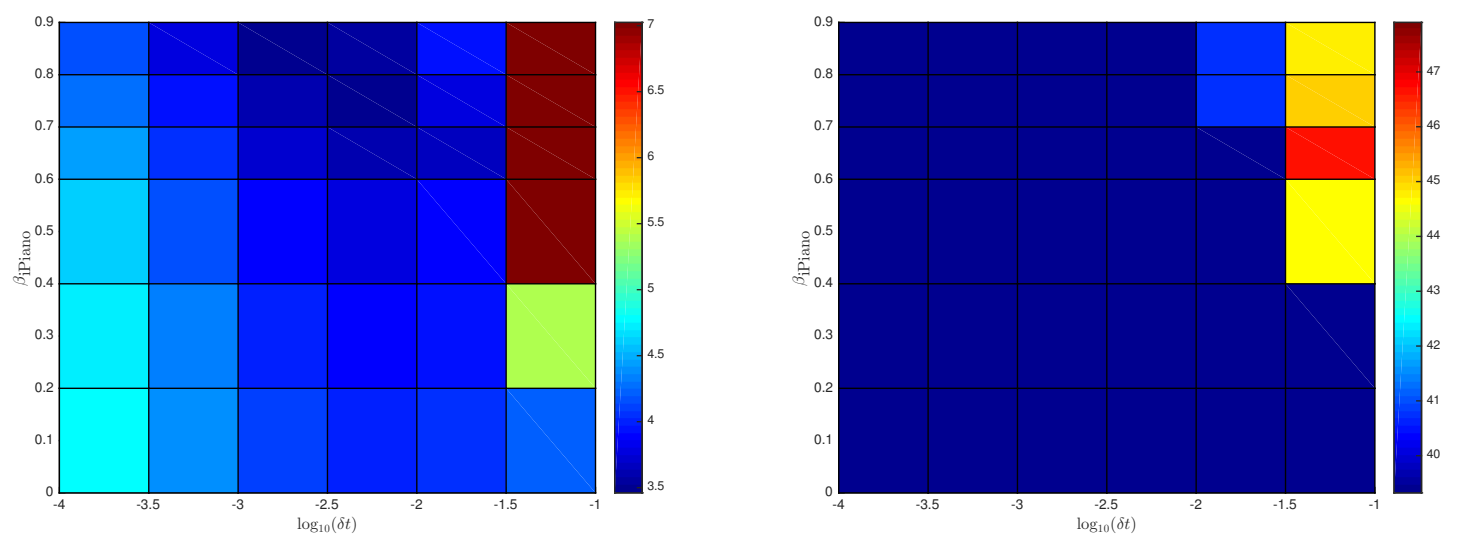

Figure 2: Influence of the parameters $\delta t$ and $\beta_{\text {iPiano on }} n_{\beta_{\text {iPiano }}}^{\text {tot }}$ (left figure, $\log _{10}$ scale) and on the energy $\mathscr{E}_{\beta, \Omega}\left(\phi^{\text {computed }}\right)$ (right figure). In both simulations, we have taken $N_{x}=2^{9}$.

energy corresponds to a local minima and not a global minima (see Figure 2 right). This property was already observed for the standard BESP scheme.

Concerning the influence of $N_{x}$, there is a minimal value to ensure that the computed solution is actually a ground state (see Figure 3 , right). In our test, this threshold value is $N_{x}=2^{8}$. This characteristic was also observed before for the classical BESP scheme. Furthermore, the optimal $\beta_{\text {iPiano }}$ parameters belongs to $(0.7,0.8)$ (see Figure 3, left) which is in good agreement with our previous results (Figure 2). As a conclusion, the choice of $\beta_{\text {iPiano }}$ mainly depends on $\delta t$.

We consider now a more confining potentiel called quadratic-plus-quartic potential, i.e.

$$
V(x)=\frac{1-\alpha}{2} x^{2}+\frac{\kappa}{4} x^{4}
$$

with $\alpha=1.2$ and $\kappa=0.3$. The numerical parameters are the same as in the harmonic case. We report $r_{\beta_{\text {iPiano }}}$ with respect to $\beta_{\text {iPiano }}$ and $\beta$ on Figure 4 (we do not plot the error $\delta \mathscr{E}_{\beta, \Omega}$ on the energy since the results are about the same as for the quadratic case). Concerning the computational cost, one gets $r_{\beta_{\text {iPiano }}} \simeq 30 \%$ as previously with the quasi-optimal value of the inertial term $\beta_{\text {iPiano }} \simeq 0.75-0.8$. The results are only weakly affected by the value of $\beta$ which means that $\operatorname{BESP}\left(\beta_{\text {iPiano }}\right)$ is relatively stable thanks to the physical/numerical parameters.

\subsection{The two-dimensional case}

We analyze now the results for the two-dimensional case for a non-rotating and next a rotating BEC. We first consider the quadratic potential: $V(\mathbf{x})=\|\mathbf{x}\|^{2} / 2$ and $\beta \in[0,3000]$. The numerical parameters 

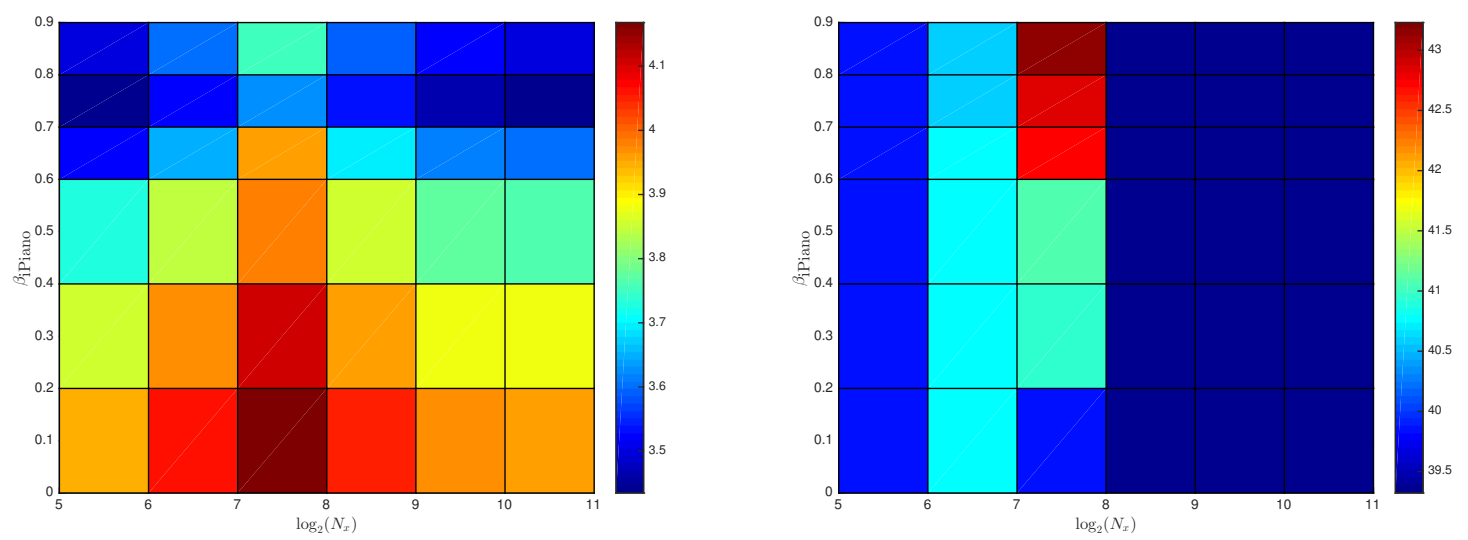

Figure 3: Influence of the parameters $N_{x}$ and $\beta_{\text {iPiano }}$ on $n_{\beta_{\text {iPian }}}^{\text {tot }}$ (right figure). In both simulations, we have taken $\delta t=10^{-2}$

(left figure, $\log _{10}$ scale) and on the energy $\mathscr{E}_{\beta, \Omega}\left(\phi^{\text {computed }}\right)$

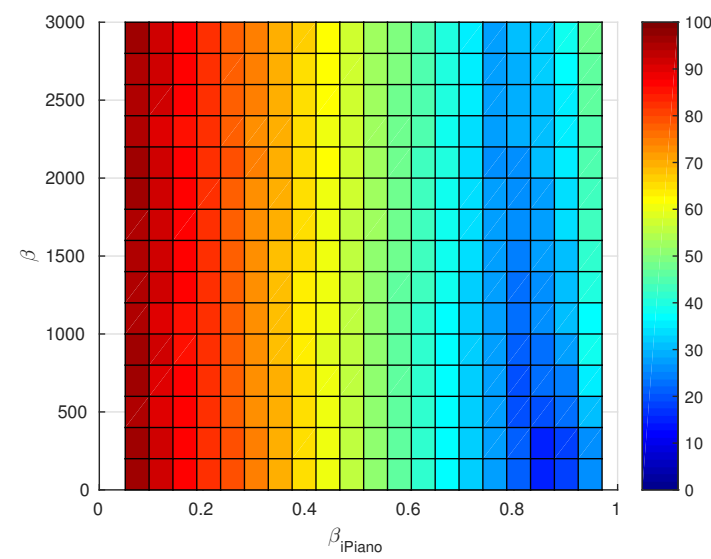

Figure 4: $\operatorname{BESP}\left(\beta_{\text {iPiano }}\right)$ for the $1 \mathrm{~d}$ quadratic-plus-quartic potential: $r_{\beta_{\text {iPiano }}}(\%)$ vs. the inertial parameter $\beta_{\text {iPiano }}$ and nonlinearity strength $\beta$.

are fixed to: $a_{x}=a_{y}=10, N_{x}=N_{y}=2^{7}, \delta t=10^{-2}$ and $\tau=10^{-10}$. From Figure 5 (left), we can see that the conclusion is very similar to the $1 \mathrm{~d}$ case. Indeed, the computational cost of the global procedure

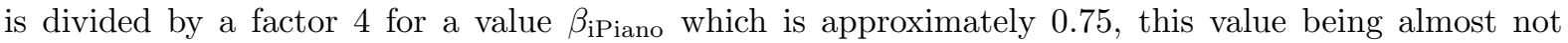
sensitive according to the nonlinearity strength $\beta$. We next perform a numerical study of the influence of the parameter $\delta t$ on the choice of the optimal value of the inertial parameter $\beta_{\text {iPiano. We do not }}$ report the influence of $N_{x} \times N_{y}$ which is weak as for the 1d case. We draw similar conclusions: optimal parameters correspond to $\left(\beta_{\text {iPiano }}^{*}, \delta t^{*}\right) \in(0.6,0.8) \times\left(10^{-3}, 10^{-2}\right)$ (see Figure 5 , right).

We consider now the optical potential

$$
V(\mathbf{x})=\frac{\|\mathbf{x}\|^{2}}{2}+25 \sin ^{2}\left(\frac{\pi}{4} x\right)+25 \sin ^{2}\left(\frac{\pi}{4} y\right) .
$$

The nonlinearity strength $\beta$ varies in $[0,3000]$. The parameters for the numerical simulation are: $a_{x}=$ $a_{y}=16, N_{x}=N_{y}=2^{7}, \delta t=10^{-2}$ and $\tau=10^{-10}$. The results presented in Figure 6 (left) show that

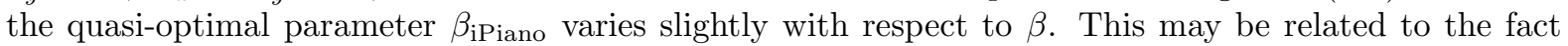
that the condensate needs to spread out in the optical lattice to reach the ground state. Nevertheless, the computational gain remains very interesting considering the small modification of an existing BESP code that is required. The range of optimal parameters $\left(\beta_{\mathrm{iPiano}}^{*}, \delta t^{*}\right)$ to get the correct energy is similar to the harmonic case (Figure6, right).

We present now the case of a two-dimensional rotating $\operatorname{GPE}(\Omega=0.5)$ with the harmonic potential and for the two nonlinearity strengths $\beta=500$ (Figure 7, top row) and 750 (Figure 7. bottom row). The parameters for the discretization/convergence are $a_{x}=a_{y}=10, N_{x}=N_{y}=2^{7}, \delta t=10^{-3}$ and 

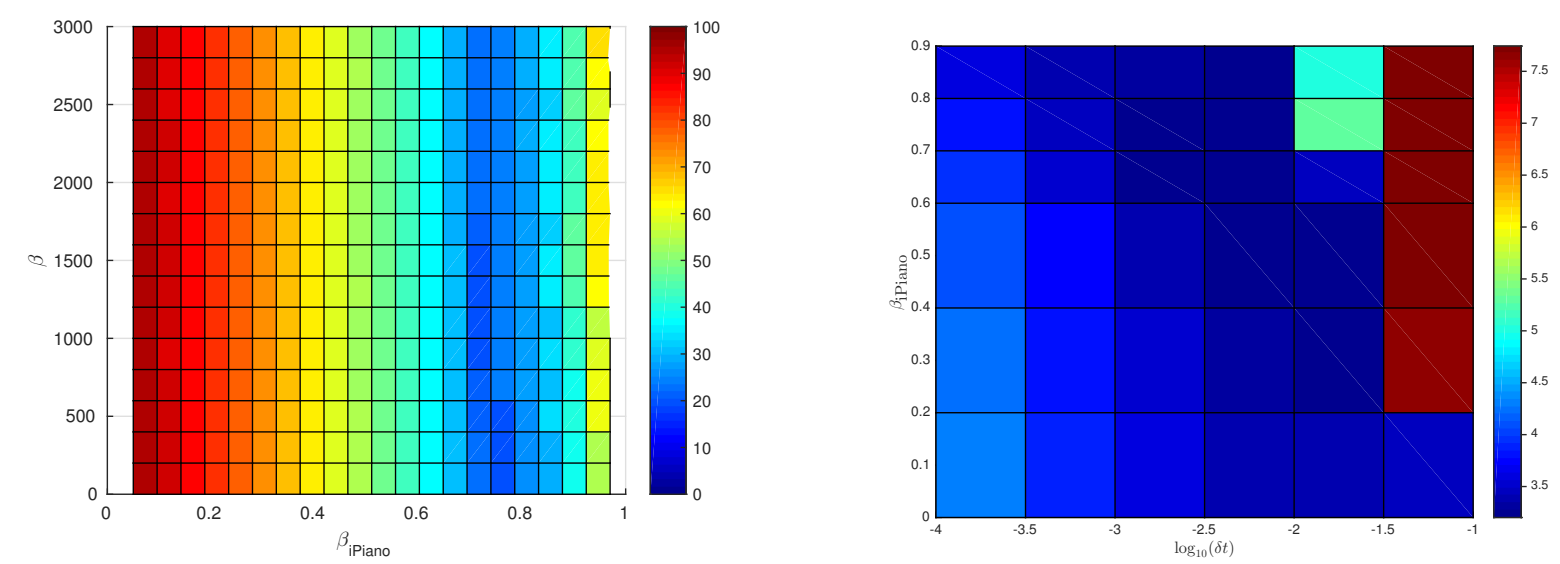

Figure 5: $\operatorname{BESP}\left(\beta_{\text {iPiano }}\right)$ for the 2 d quadratic potential $(\Omega=0): r_{\beta_{\text {Piano }}}($ left, $\%)$ and energy $\mathscr{E}_{\beta, \Omega}\left(\phi^{\text {computed }}\right)$ (right) vs. the inertial parameter $\beta_{\mathrm{iPiano}}$ and nonlinearity strength $\beta$.
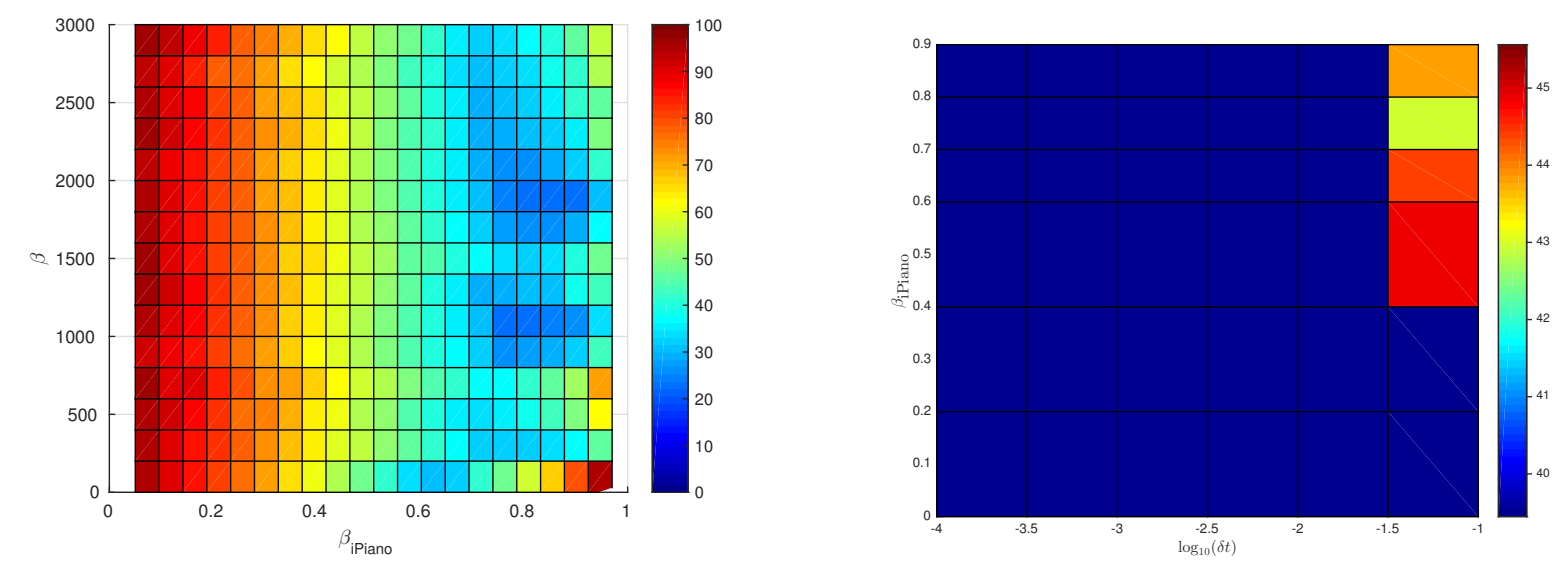

Figure 6: $\operatorname{BESP}\left(\beta_{\text {iPiano }}\right)$ for the 2 d quadratic-plus-quartic potential $(\Omega=0): r_{\beta_{\text {iPiano }}}$ (left, \%) and energy $\mathscr{E}_{\beta, \Omega}\left(\phi^{\text {computed }}\right)$ (right) vs. the inertial parameter $\beta_{\mathrm{iPiano}}$ and nonlinearity strength $\beta$.

$\tau=10^{-6}$. We report in Fig. 7 the results concerning the computed energy $\mathscr{E}_{\beta, \Omega}\left(\phi^{\text {computed }}\right)$ (left column, red line, the blue line being the energy for BESP with the same discretization parameters) and $r_{\beta_{\mathrm{P} \text { Piano }}}$ (right column) vs. $\beta_{\text {iPiano }}$ for the two values of $\beta$. In this case, a quasi-optimal value of $\beta_{\text {iPiano is rather }}$ $0.8-0.85$ and the associated gain is about $70 \%$ of the whole computational time. We remark that for values of $\beta_{\text {iPiano }}$ close to 1 , the computed energy is inaccurate (e.g. the energy for $\beta_{\text {iPiano }}=0.97$ is 8.02800836), otherwise the value of the energy for $\operatorname{BESP}\left(\beta_{\mathrm{iPiano}}\right)$ is less than the one for BESP.

\subsection{The three-dimensional case}

We report now in Figure 8 the results for the three-dimensional quadratic case, i.e. $V(\mathbf{x})=\|\mathbf{x}\|^{2} / 2$, without rotation $(\Omega=0)$ and for $\beta=100$. The computational domain is $]-10,10{ }^{3}$ which is discretized with $2^{6}$ Fourier modes in each direction. The time step is $\delta t=10^{-2}$ and $\tau=10^{-6}$. The quasi-optimal value of $\beta_{\mathrm{iPiano}}$ is 0.8 which leads to a gain of $80 \%$ of the computational time compared with BESP. This gain is very interesting since each iteration is quite expensive in the three-dimensional situation. As we can see, the computed energy of $\operatorname{BESP}\left(\beta_{\text {iPiano }}\right)$ is correct for $0 \leq \beta_{\text {iPiano }} \leq 0.85$ but may be incorrect for values of $\beta_{\text {iPiano }}$ too close to 1 .

To end the paper, we present some results for a GPE with a rotation term $\Omega=0.7$ and with a quadratic potential $V$. In addition, the GPE includes a dipolar interaction term

$$
\left(d^{2} \int_{\mathbb{R}^{3}} \frac{1-3 \cos ^{2} \widehat{(\mathbf{a}, \tilde{\mathbf{x}})}}{\|\mathbf{x}-\tilde{\mathbf{x}}\|^{3}}|\phi(t, \tilde{\mathbf{x}})|^{2} d \tilde{\mathbf{x}}\right) \phi(t, \mathbf{x}),
$$



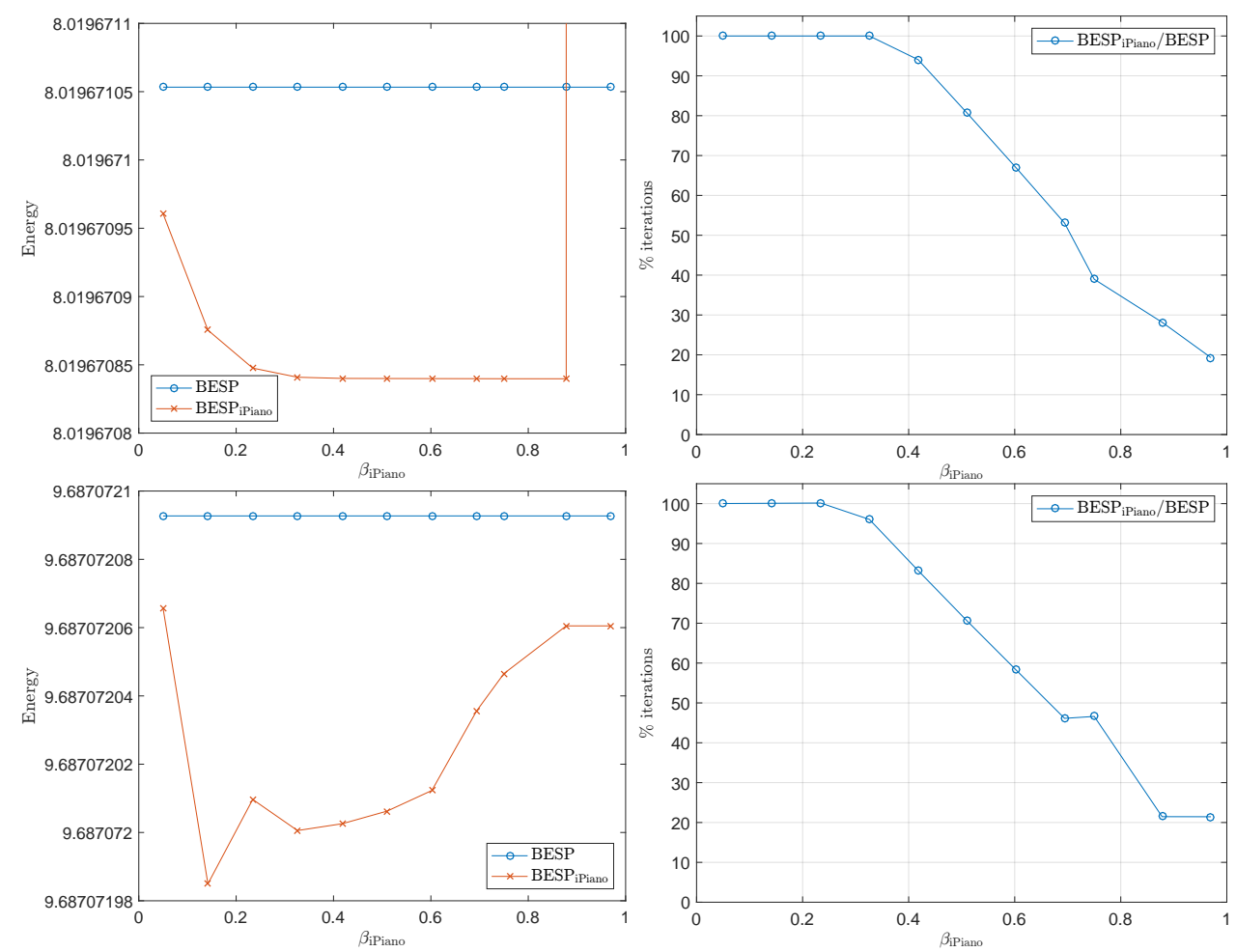

Figure 7: $\operatorname{BESP}\left(\beta_{\text {iPiano }}\right)$ for the $2 \mathrm{~d}$ quadratic potential and with $\Omega=0.5$ (top: $\beta=500 ;$ bottom $\beta=750$ ): energy $\mathscr{E}_{\beta, \Omega}\left(\phi^{\text {computed }}\right)$ (left) and $r_{\beta_{\text {iPiano }}}$ (right, \%) vs. $\beta_{\text {iPiano. }}$.
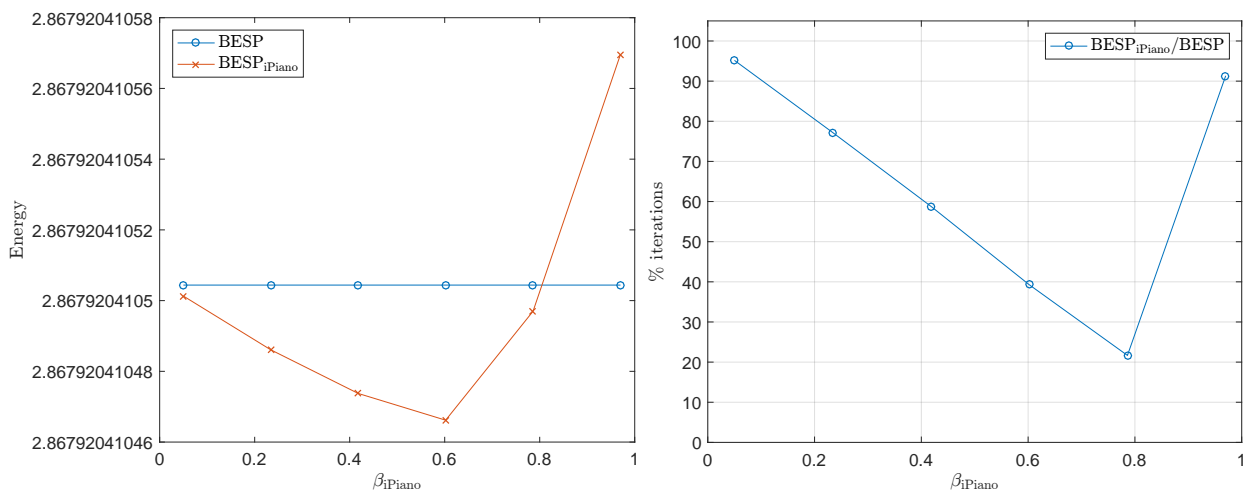

Figure 8: $\operatorname{BESP}\left(\beta_{\text {iPiano }}\right)$ for the $3 \mathrm{~d}$ quadratic potential $(\Omega=0)$ and $\beta=100$ : energy $\mathscr{E}_{\beta, \Omega}\left(\phi^{\text {computed }}\right)$ (left) and $r_{\beta_{\text {iPiano }}}$ (right, $\%$ ) vs. $\beta_{\text {iPiano }}$.

added to the cubic nonlinearity. The physical parameters are $\beta=500, d=0.5$ and $a={ }^{t}(0,0,1)$. The computational domain is $]-10,10\left[{ }^{3}\right.$ discretized with $2^{7}$ Fourier modes in each direction. The time step is $\delta t=10^{-2}$ and $\tau=10^{-6}$. We consider $\operatorname{BESP}\left(\beta_{\text {iPiano }}=0.7\right)$ which requires $N^{\text {out }}=2973$ iterations (for $n_{\beta_{\mathrm{iPiano}}}^{\text {tot }}=27503$ BiCGStab iterations) compared with $\operatorname{BESP}(=\operatorname{BESP}(0))$ which needs $N^{\text {out }}=6396$ iterations (for $n_{0}^{\text {tot }}=59250$ BiCGStab iterations). The computed energy is equal to 5.374118. We draw on Figure 9 the $10^{-3.5}$ and $10^{-3}$ isovalues of $|\phi(t, \mathbf{x})|^{2}$. We present on Figure 10 the $(x, y)$-slice of $|\phi(t, \mathbf{x})|^{2}$ and of the angle of $\phi(t, \mathbf{x})$ showing the placement of the vortex lines.

\section{Conclusion}

The aim of this paper was to introduce and test a slight modification of the standard BESP scheme for spectrally computing the stationary states of GPEs that model BEC. The new scheme, called $\operatorname{BESP}\left(\beta_{\mathrm{iPiano}}\right)$, is based on an implicit version of the iPiano optimization algorithm recently introduced in [35] for image processing, combined with FFT pseudospectral approximation schemes in space. 
$\Omega=0.7, \beta=500$

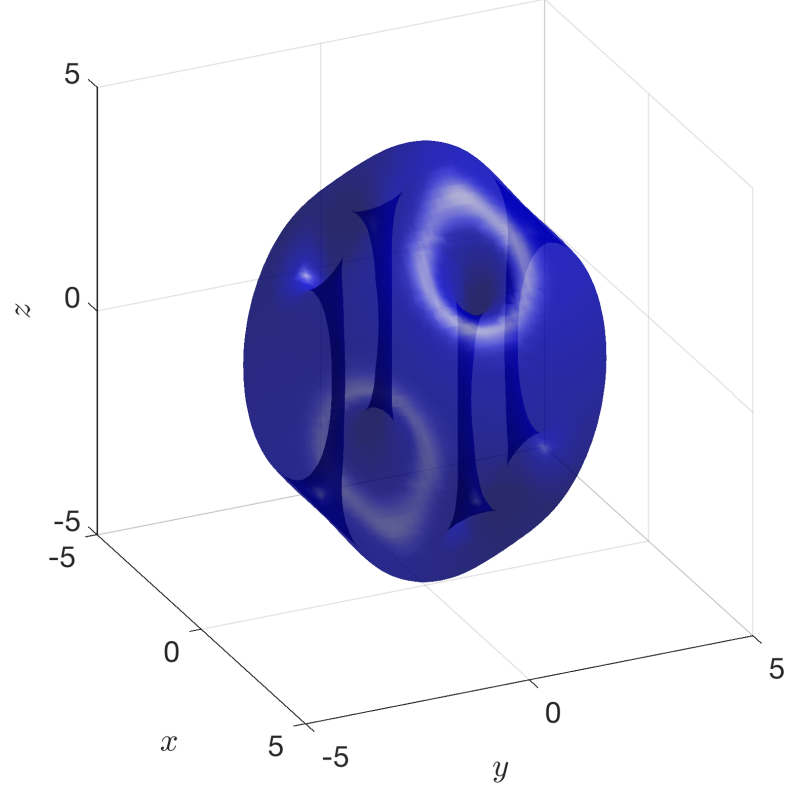

$\Omega=0.7, \beta=500$

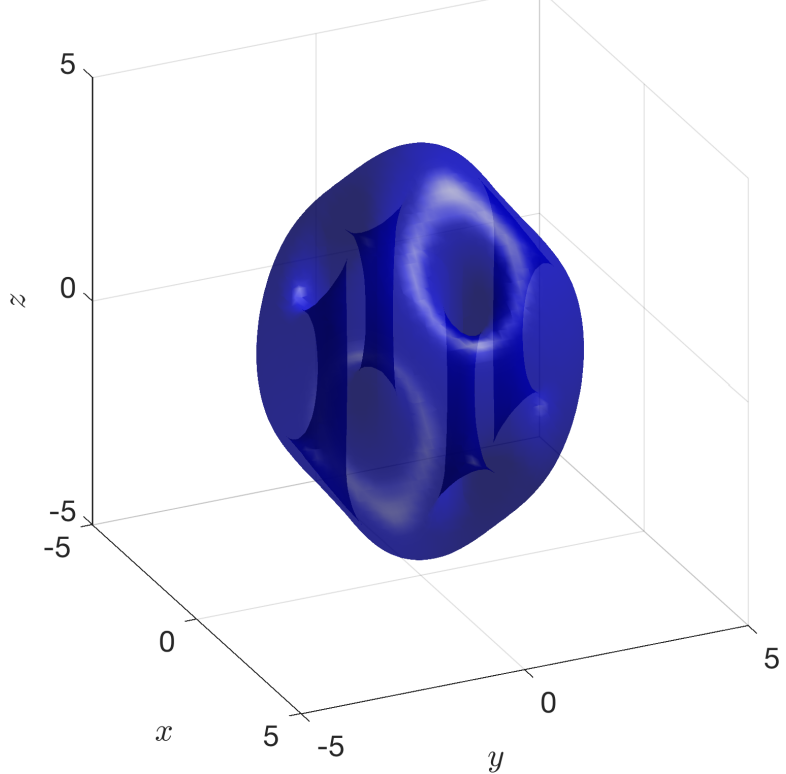

Figure 9: $10^{-3.5}$ (left) and $10^{-3}$ (right) isovalues of $|\phi(t, \mathbf{x})|^{2}$.
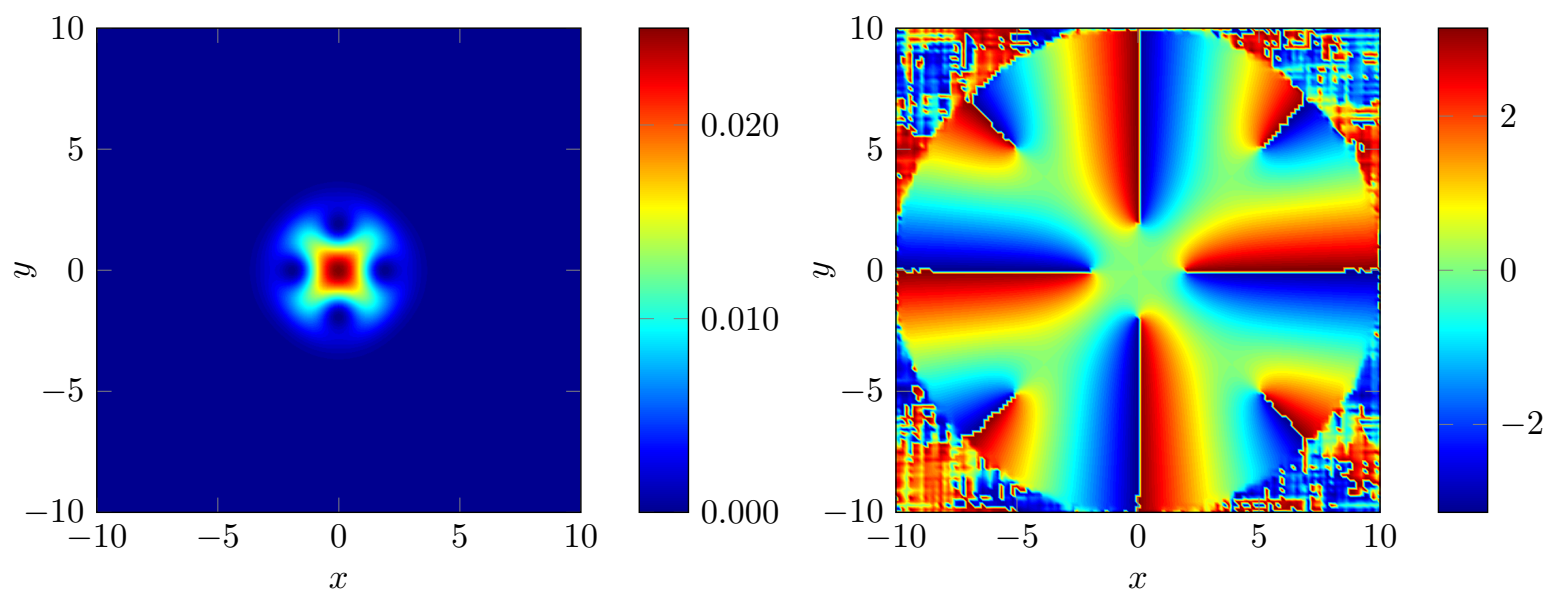

Figure 10: $(x, y)$-slice of $|\phi(t, \mathbf{x})|^{2}$ (left) and of the angle of $\phi$ (right).

While the new algorithm can be trivially integrated in an existing BESP code, the global computational cost of the standard BESP algorithm (e.g. $\beta_{\text {iPiano }}=0$ ) is divided by a factor 3 to 4 . To validate the method, we reported a thorough numerical study for 1d-2d-3d GPEs, with possible rotation and dipolar interaction. We then conclude that an nearly optimal value of $\beta_{\text {iPiano }}$ is $0.75-0.8$, and weakly depends on the problem parameters.

Acknowledgments. The authors thank the support of the french ANR grant ANR-12-MONU-0007-02 BECASIM (Modèles Numériques call).

\section{References.}

[1] J.R. Abo-Shaeer, C. Raman, J.M. Vogels, and W. Ketterle, Observation of vortex lattices in Bose-Einstein condensates, Science 292 (2001), no. 5516, 476-479.

[2] S.K. Adhikari, Numerical solution of the two-dimensional Gross-Pitaevskii equation for trapped interacting atoms, Physics Letters A 265 (2000), no. 1-2, 91-96.

[3] A. Aftalion and Q. Du, Vortices in a rotating Bose-Einstein condensate: Critical angular velocities and energy diagrams in the Thomas-Fermi regime, Physical Review A 64 (2001), no. 6, 063603.

[4] M.H. Anderson, J.R. Ensher, M.R. Matthews, C.E. Wieman, and E.A. Cornell, Observation of Bose-Einstein condensation in a dilute atomic vapor, Science 269 (1995), no. 5221, 198-201. 
[5] X. Antoine and R. Duboscq, GPELab, a Matlab toolbox to solve Gross-Pitaevskii equations I: Computation of stationary solutions, Computer Physics Communications 185 (2014), no. 11, 2969-2991.

[6] _ Robust and efficient preconditioned Krylov spectral solvers for computing the ground states of fast rotating and strongly interacting Bose-Einstein condensates, Journal of Computational Physics 258 (2014), $509-523$.

[7] _ GPELab, a Matlab toolbox to solve Gross-Pitaevskii equations II: Dynamics and stochastic simulations, Computer Physics Communications 193 (2015), 95-117.

[8] — Modeling and Computation of Bose-Einstein Condensates: Stationary States, Nucleation, Dynamics, Stochasticity, Nonlinear Optical and Atomic Systems: at the Interface of Physics and Mathematics (Besse, C and Garreau, JC, ed.), Lecture Notes in Mathematics, vol. 2146, 2015, pp. 49-145.

[9] W. Bao, Ground states and dynamics of multi-component Bose-Einstein condensates, Multiscale Modeling and Simulation: A SIAM Interdisciplinary Journal 2 (2004), no. 2, 210-236.

[10] W. Bao and Y. Cai, Ground states of two-component Bose-Einstein condensates with an internal atomic Josephson junction, East Asian Journal on Applied Mathematics 1 (2011), 49-81.

[11] _ Mathematical theory and numerical methods for Bose-Einstein condensation, Kinetic and Related Models 6 (2013), no. 1, 1-135.

[12] W. Bao, Y. Cai, and H. Wang, Efficient numerical methods for computing ground states and dynamics of dipolar Bose-Einstein condensates, Journal of Computational Physics 229 (2010), no. , 7874-7892.

[13] W.Z. Bao and Q. Du, Computing the ground state solution of Bose-Einstein condensates by a normalized gradient flow, SIAM Journal on Scientific Computing 25 (2004), no. 5, 1674-1697.

[14] W.Z. Bao and W.J. Tang, Ground-state solution of Bose-Einstein condensate by directly minimizing the energy functional, Journal of Computational Physics 187 (2003), no. 1, 230-254.

[15] D. Baye and J.M. Sparenberg, Resolution of the Gross-Pitaevskii equation with the imaginary-time method on a Lagrange mesh, Physical Review E 82 (2010), no. 5, 2.

[16] C.C. Bradley, C.A. Sackett, J.J. Tollett, and R.G. Hulet, Evidence of Bose-Einstein condensation in an atomic gas with attractive interactions, Physical Review Letters 75 (1995), no. 9, 1687-1690.

[17] V. Bretin, S. Stock, Y. Seurin, and J. Dalibard, Fast rotation of a Bose-Einstein condensate, Physical Review Letters 92 (2004), no. 5.

[18] M. Caliari, A. Ostermann, S. Rainer, and M. Thalhammer, A minimisation approach for computing the ground state of Gross-Pitaevskii systems, Journal of Computational Physics 228 (2009), no. 2, 349-360.

[19] M. Caliari and S. Rainer, GSGPEs: A MATLAB code for computing the ground state of systems of Gross-Pitaevskii equations, Computer Physics Communications 184 (2013), no. 3, 812-823.

[20] E. Candès and B. O'Donoghue, Adaptive Restart for Accelerated Gradient Schemes, Foundations of Computational Mathematics 15 (2013), no. 3, 715-732.

[21] M.M. Cerimele, M.L. Chiofalo, F. Pistella, S. Succi, and M.P. Tosi, Numerical solution of the Gross-Pitaevskii equation using an explicit finite-difference scheme: An application to trapped Bose-Einstein condensates, Physical Review E 62 (2000), no. 1, B, 1382-1389.

[22] M.L. Chiofalo, S. Succi, and M.P. Tosi, Ground state of trapped interacting Bose-Einstein condensates by an explicit imaginary-time algorithm, Physical Review E 62 (2000), no. 5, B, 7438-7444.

[23] F. Dalfovo, S. Giorgini, L.P. Pitaevskii, and S. Stringari, Theory of Bose-Einstein condensation in trapped gases, Review of Modern Physics 71 (1999), no. 3, 463-512.

[24] I. Danaila and F. Hecht, A finite element method with mesh adaptivity for computing vortex states in fast-rotating Bose-Einstein condensates, Journal of Computational Physics 229 (2010), no. 19, 6946-6960.

[25] I. Danaila and P. Kazemi, A new Sobolev gradient method for direct minimization fo the Gross-Pitaevskii energy with rotation, SIAM Journal on Scientific Computing 32 (2010), no. 5, 2447-2467.

[26] K.B. David, M.O. Mewes, M.R. Andrews, N.J. Vandruten, D.S. Durfee, D.M. Kurn, and W. Ketterle, Bose-Einstein Condensation in gas of sodium atoms, Physical Review Letters 75 (1995), no. 22, 3969-3973.

[27] C.M. Dion and E. Cances, Ground state of the time-independent Gross-Pitaevskii equation, Computer Physics Communications 177 (2007), no. 10, 787-798.

[28] M. Edwards and K. Burnett, Numerical solution of the nonlinear Schrödinger equation for small samples of trapped neutral atoms, Physical Review A 51 (1995), no. 2, 1382-1386.

[29] A. Gammal, T. Frederico, and L. Tomio, Improved numerical approach for the time-independent Gross-Pitaevskii nonlinear Schrödinger equation, Physical Review E 60 (1999), no. 2, B, 2421-2424.

[30] K.W. Madison, F. Chevy, V. Bretin, and J. Dalibard, Stationary states of a rotating Bose-Einstein condensate: Routes to vortex nucleation, Physical Review Letters 86 (2001), no. 20, 4443-4446.

[31] K.W. Madison, F. Chevy, W. Wohlleben, and J. Dalibard, Vortex formation in a stirred Bose-Einstein condensate, Physical Review Letters 84 (2000), no. 5, 806-809.

[32] M.R. Matthews, B.P. Anderson, P.C. Haljan, D.S. Hall, C.E. Wieman, and E.A. Cornell, Vortices in a Bose-Einstein condensate, Physical Review Letters 83 (1999), no. 13, 2498-2501.

[33] Y. Nesterov, A method for unconstrained convex minimization problem with the rate of convergence O (1/k2), Doklady an SSSR, vol. 269, 1983, pp. 543-547.

[34] Introductory lectures on convex optimization, vol. 87, Springer Science \& Business Media, 2004.

[35] P. Ochs, Y. Chen, T. Brox, and T. Pock, iPiano: Inertial proximal algorithm for nonconvex optimization, SIAM Journal on Imaging Sciences 7 (2014), no. 2, 1388-1419.

[36] C. Raman, J.R. Abo-Shaeer, J.M. Vogels, K. Xu, and W. Ketterle, Vortex nucleation in a stirred Bose-Einstein condensate, Physical Review Letters 87 (2001), no. 21.

[37] Y.-S. Wang and C.-S. Chien, A spectral-Galerkin continuation method using Chebyshev polynomials for the numerical solutions of the Gross-Pitaevskii equation, Journal of computational and applied mathematics 235 (2011), no. 8, $2740-2757$.

[38] Y.-S. Wang, B.-W. Jeng, and C.-S. Chien, A Two-Parameter Continuation Method for Rotating Two-Component Bose-Einstein Condensates in Optical Lattices, Communications in Computational Physics 13 (2013), $442-460$.

[39] X. Wu, Z. Wen, and W. Bao, A regularized Newton method for computing ground states of Bose-Einstein condensates, 
submitted, arxiv: 1504.02891, 21 pages.

[40] C. Yuce and Z. Oztas, Off-axis vortex in a rotating dipolar Bose-Einstein condensate, Journal of Physics B-Atomic Molecular and Optical Physics 43 (2010), no. 13.

[41] R. Zeng and Y. Zhang, Efficiently computing vortex lattices in rapid rotating Bose-Einstein condensates, Computer Physics Communications 180 (2009), no. 6, 854-860. 